\title{
ENERGY EFFICIENCY OF CONSECUTIVE FRAGMENTATION PROCESSES
}

\author{
JOAQUÍN FONTBONA, ${ }^{* *}$ Universidad de Chile \\ NATHALIE KRELL, ${ }^{* * *}$ Université Rennes 1 \\ SERVET MARTÍNEZ, ${ }^{* * * *}$ Universidad de Chile
}

\begin{abstract}
Motivated by a problem arising in the mining industry, we present a first study of the energy required to reduce a unit mass fragment by consecutively using several devices. Two devices are considered, which we represent as different stochastic fragmentation processes. Following the self-similar energy model introduced in Bertoin and Martínez (2005), we compute the average energy required to attain a size $\eta_{0}$ with this two-device procedure. We then asymptotically compare, as $\eta_{0}$ goes to 0 or 1 , its energy requirement with that of individual fragmentation processes. In particular, we show that, for a certain range of parameters of the fragmentation processes and of their energy cost functions, the consecutive use of two devices can be asymptotically more efficient than using each of them separately, or vice versa.
\end{abstract}

Keywords: Fragmentation process; fragmentation energy; subordinator; Laplace exponent

2010 Mathematics Subject Classification: Primary 60J85

$$
\text { Secondary } 60 \mathrm{~J} 80
$$

\section{Introduction}

The present work is motivated by the mining industry, where mechanical devices are used to break rocks in order to liberate the metal contained in them. This fragmentation procedure is carried out in a series of steps (the first of them being blasting, followed then by crushers, grinders, or mills) until fragments attain a sufficiently small size for mining purposes. One of the problems that faces the mining industry is to minimize the total amount of energy consumed in this process. To be more precise, at each intermediate step, material is broken by a repetitive mechanism until particles can go across a classifying grid and onto the next step. The output sizes are known not to be optimal in terms of the global energy cost. Moreover, since crushers or mills are large machines and seldom replaced, the output sizes are in practice one of the few parameters on which a decision can be made.

In an idealized setting, the problem might be posed as follows. Suppose that a unit-sized fragment is reduced to fragments of size less than a fixed threshold $\eta_{0} \in(0,1]$ by passing the fragment through two different consecutive fragmentation mechanisms (for instance, the first being crushers and the second being mills). In this 'two-step' fragmentation procedure,

\footnotetext{
Received 23 November 2009; revision received 19 March 2010.

* Postal address: Departamento de Ingeniería Matemática and Centro de Modelamiento Matemático, Universidad de Chile, UMI 2807 CNRS, Casilla 170-3, Correo 3, Santiago, Chile.

** Email address: fontbona@ dim.uchile.cl

*** Postal address: Institut de Recherche Mathématique de Rennes (IRMAR), Université Rennes 1, UMR 6625 CNRS, Campus de Beaulieu, 35042 Rennes Cedex, France. Email address: nathalie.krell@univ-rennes1.fr

**** Email address: smartine@dim.uchile.cl
} 
each mass fragment evolves in the first fragmentation mechanism until it becomes smaller than $\eta \in\left(\eta_{0}, 1\right]$, at which point it immediately enters the second mechanism. Then, the fragment continues to evolve until the first instant it becomes smaller than $\eta_{0}$, when it finally exits the system. The central question is:

(A) What is the optimal choice for the intermediate threshold $\eta$ ?

To formulate this problem, we will model each fragmentation mechanism by a continuoustime random fragmentation process, in which particles break independently of each other (branching property) and in a self-similar way. (For a recent account and developments in the mathematical theory of fragmentation processes, we refer the reader to [5]. The self-similarity hypothesis agrees with observations made in the mining industry; see, e.g. [10, p. 255]. In particular, it is reasonable to assume that the energy required to break a block of size $s$ into a set of smaller blocks of size $\left(s_{1}, s_{2}, \ldots\right)$ is of the form $s^{\beta} \varphi\left(s_{1} / s, s_{2} / s, \ldots\right)$, where $\varphi$ is a cost function and $\beta>0$ is a fixed parameter. For example, in the so-called potential case, we have $\varphi\left(s_{1}, s_{2}, \ldots\right)=\sum_{n=1}^{\infty} s_{n}^{\beta}-1$, which corresponds to the law of Charles, Walker, and Bond [10].

Within this mathematical framework, the asymptotic behavior of the energy required by a single fragmentation process to reduce all fragments to sizes less than $\eta$ was studied in [6]. It was shown that the mean energy behaves as $1 / \eta^{\alpha-\beta}$ when $\eta \rightarrow 0$, where $\alpha$ denotes the Malthusian exponent of the fragmentation process and $\alpha>\beta$ in physically reasonable cases. Therefore, the performances of two individual fragmentation processes are asymptotically comparable by means of the quantities $\alpha-\beta$ and $\hat{\alpha}-\hat{\beta}$, where $\hat{\alpha}>\hat{\beta}$ are the parameters associated with a second fragmentation process.

We will formulate problem (A) in mathematical terms, adopting the same mean energy point of view as in [6]. First, we will explicitly compute the objective function, which we express in terms of the Lévy and renewal measures associated with the 'tagged fragment' of each of the two fragmentation processes (see [4]). Then, our goal will be to study a preliminary question related to (A), which is weaker but still relevant to the mining industry:

(B) Is the above described 'two-step' procedure efficient in terms of the mean energy, compared to the 'one-step' procedures where only the first or second fragmentation mechanism reduces a unit-size fragment to fragments not larger than $\eta_{0}$ ?

We will address this question in asymptotic regimes, namely for $\eta$ and $\eta_{0}$ going together either to 0 or to 1 . In both cases, we will give explicit estimates in terms of $\eta$ for the efficiency gain or loss of using the two-step procedure.

As we will see, if $\alpha, \beta, \hat{\alpha}$, and $\hat{\beta}$ are different, for any values of $\eta / \eta_{0} \in(0,1)$, the relations between these four parameters determine the relative efficiency between the first, the second, and the two-step fragmentation procedures if $\eta$ is sufficiently small. In particular, when $\alpha>\hat{\alpha}$ and $\beta>\hat{\beta}$, the answer to (B) is affirmative for sufficiently small $\eta$, so that the solution to problem $(\mathrm{A})$ is in general nontrivial.

We will carry out a similar analysis for large (that is, close to unit-size) thresholds. In order to quantify the comparative efficiency of the two-step procedure, we will make an additional hypothesis of regular variation at $\infty$ of the Lévy exponents of the tagged fragment processes. This will be transparently interpreted in terms of the infinitesimal average energy required by each of the fragmentation processes to break arbitrarily close to unit-size fragments. We will show that, at least for small values of $\log \eta_{0} / \log \eta$ and variation indices in $\left(0, \frac{1}{2}\right]$ for both fragmentation processes, the relative infinitesimal efficiency of the two fragmentation processes determines the comparative efficiency of the three alternative fragmentation procedures if $\eta$ is sufficiently close to 1 . 
We point out that the relevant parameters involved in our analysis could in principle be statistically estimated. A first concrete step in this direction has been made by Hoffmann and Krell [8], who asymptotically estimated the Lévy measure of the tagged fragment from the observations of the sizes of fragments at the instant they become smaller than $\eta \rightarrow 0$. Although this is in general not enough to recover the characteristics of the fragmentation process, it provides all the relevant parameters we need which are not observable by other means.

The remainder of this paper is organized as follows. In Section 2 we recall the construction of homogeneous fragmentation processes in terms of Poisson point processes, we describe our model of the two-step fragmentation procedure and compute its average energy using first passage laws for subordinators. In Section 3 we recall some results on renewal theory for subordinators and use them to study the small threshold asymptotics of our problem in Theorems 1 and 2, where the two-step procedure is respectively compared with the first and second fragmentation processes. The comparative efficiency of the three alternatives according to the values of $\alpha, \beta, \hat{\alpha}$, and $\hat{\beta}$ is summarized in Corollary 1 . In Section 4 we introduce the idea of relative 'infinitesimal efficiency' of two fragmentation procedures. We relate it to a regular variation assumption at $\infty$ for the Lévy exponent of the tagged fragment, and use it to analyze the comparative efficiency of the two-step fragmentation procedure for close to unit-size fragments, using Dynkin-Lamperti asymptotics for subordinators at first passage.

\section{The model}

\subsection{The fragmentation process}

We will model the fragmentation mechanisms as a homogeneous fragmentation process, as introduced in [4]. This is a homogeneous Markov process $\boldsymbol{X}=(X(t, \boldsymbol{x}): t \geq 0)$ taking values in

$$
s^{\downarrow}:=\left\{s=\left(s_{1}, s_{2}, \ldots\right): s_{1} \geq s_{2} \geq \cdots \geq 0, \sum_{i=1}^{\infty} s_{i} \leq 1\right\},
$$

where the parameter $\boldsymbol{x}=\left(x_{1}, x_{2}, \ldots\right) \in s^{\downarrow}$ stands for the initial condition (i.e. $X(0, \boldsymbol{x})=\boldsymbol{x}$ almost surely), and which satisfies the two fundamental properties of branching and homogeneity. More precisely, different fragments of $X(t, \boldsymbol{x})$ split up independently of one another (branching) and, denoting by $X(t)=X(t, \boldsymbol{x})$ the process started from $\boldsymbol{x}=(1,0, \ldots)$, it holds that $r X(t)$ has the same law as $X(t,(r, 0, \ldots))$ for any $r \in[0,1]$ (homogeneity).

We observe that homogeneous fragmentation processes are self-similar fragmentation processes with zero index of self-similarity (see [5, Chapter 2]). Since self-similar fragmentation processes with different indices are related by a family of random time changes (depending on the fragments), there is no loss of generality in working here in the homogeneous case as the quantities we study are only size dependent (see also [6]).

We assume that no creation of mass occurs. It is known that in this case, the process $\boldsymbol{X}$ is entirely characterized by an erosion coefficient $c \geq 0$ and a dislocation measure $v$, which is a measure on $s \downarrow$ satisfying the conditions

$$
v(\{1,0,0, \ldots\})=0 \text { and } \int_{\delta \downarrow}\left(1-s_{1}\right) v(\mathrm{~d} s)<\infty .
$$

Moreover, we suppose that we are in the dissipative case, $\sum_{i=1}^{\infty} s_{i} \leq 1$ almost surely, and we assume absence of erosion, i.e. $c=0$.

Let us recall the construction of a homogeneous fragmentation process in this setting, in terms of the atoms of a Poisson point process (see [1]). Let $v$ be a dislocation measure fulfilling 
conditions (1). Let $\boldsymbol{K}=((\Delta(t), k(t)): t \geq 0)$ be a Poisson point process with values in $s^{\downarrow} \times \mathbb{N}$, and with intensity measure $v \otimes \sharp$, where $\sharp$ is the counting measure on $\mathbb{N}$. As in [1], we can construct a unique $s^{\downarrow}$-valued process $X=(X(t, \boldsymbol{x}): t \geq 0)$ started from $\boldsymbol{x}$ with paths that jump only at those times $t \geq 0$ at which a point $\left(\Delta(t)=\left(\Delta_{1}, \Delta_{2}, \ldots\right), k(t)\right)$ occurs. More precisely, $X(t, \boldsymbol{x})$ is obtained by replacing the $k(t)$ th term $X(t-, \boldsymbol{x})$ with a decreasing rearrangement of the sequence $X_{1}(t-, \boldsymbol{x}), \ldots, X_{k-1}(t-, \boldsymbol{x}), X_{k}(t-, \boldsymbol{x}) \Delta_{1}, X_{k}(t-, \boldsymbol{x}) \Delta_{2}, \ldots$, $X_{k+1}(t-, \boldsymbol{x}), \ldots$

Define

$$
\underline{p}:=\inf \left\{p \in \mathbb{R}: \int_{s \downarrow} \sum_{j=2}^{\infty} s_{j}^{p} v(\mathrm{~d} s)<\infty\right\},
$$

and, for every $q \in(\underline{p}, \infty)$, consider

$$
\kappa(q):=\int_{\delta \downarrow}\left(1-\sum_{j=1}^{\infty} s_{j}^{q}\right) v(\mathrm{~d} s) .
$$

In the sequel, we assume that the Malthusian hypothesis holds, that is, there exists an $\alpha \geq \underline{p}$, called the Malthusian exponent, such that $\kappa(\alpha)=0$.

A key tool in fragmentation theory is the tagged fragment process $\chi=(\chi(t): t \geq 0)$ associated with $\boldsymbol{X}$. We recall that it satisfies

$$
\chi(t)=X_{J(t)}(t)
$$

where $J(t)$ is a random integer such that, conditioned on $X(t), \mathrm{P}(J(t)=i \mid X(t))=X_{i}(t)$ for all $i \geq 1$, and $\mathrm{P}(J(t)=0 \mid X(t))=1-\sum_{i=1}^{\infty} X_{i}(t)$. Moreover, as shown by Bertoin [4, Theorem 3], the process

$$
\xi_{t}=-\log \chi(t)
$$

is a subordinator whose Laplace exponent $\phi$ is given by

$$
\phi(q):=\kappa(q+1)
$$

for $q>p-1$. For a precise definition, we refer the reader to [4] or Chapter 2 of [5]. We note that the hypothesis of absence of erosion, $c=0$, implies that $\phi$ has no drift coefficient.

Since $\phi(\alpha-1)=0$, the process $\mathrm{e}^{(1-\alpha) \xi(t)}$ is a nonnegative martingale, and we can then define a probability measure $\tilde{\mathrm{P}}$ on the path space by

$$
\left.\mathrm{d} \tilde{\mathrm{P}}\right|_{\mathcal{F}_{t}}=\left.\mathrm{e}^{(1-\alpha) \xi(t)} \mathrm{dP}\right|_{\mathscr{F}_{t}},
$$

where $\left(\widetilde{F}_{t}: t \geq 0\right)$ denotes the natural filtration of $\xi$. It is well known that, under this 'tilted' law, $\xi$ is a subordinator with Laplace exponent

$$
\tilde{\phi}(q)=\phi(q+\alpha-1) .
$$

Since $\lim _{q \rightarrow \infty} \phi(q) / q=c$ (see [5, Proposition 2, Chapter I]), the previous relation and the assumption of nonerosion imply that $\tilde{\phi}$ has no drift coefficient.

We will respectively denote by $\Pi$ and $U$ the Lévy measure and the renewal measure of $\xi_{t}$ under $\tilde{\mathrm{P}}$ (see, e.g. [2, pp. 3, 74]). We recall that, for a Borel subset $A$ of $[0, \infty)$,

$$
U(A)=\int_{0}^{\infty} \tilde{\mathrm{P}}(\xi(t) \in A) \mathrm{d} t .
$$


As usual, and when there is no possible confusion, we will denote by $U(x):=U([0, x]), x \geq 0$, the distribution function associated to $U$.

For $\eta \in(0,1]$, we denote by

$$
T_{\eta}:=\inf \left\{t \geq 0: \xi_{t}>\log \left(\frac{1}{\eta}\right)\right\}
$$

the first time that the size of the tagged fragment is less than $\eta$.

\subsection{The fragmentation energy}

Following [6], we will assume that the energy needed to split a fragment of size $x \in[0,1]$ into smaller fragments of sizes $x_{1} \geq x_{2} \geq \cdots$ is given by

$$
x^{\beta} \varphi\left(\frac{x_{1}}{x}, \frac{x_{2}}{x}, \ldots\right),
$$

where $\beta>0$ is a fixed constant and $\varphi: \delta \rightarrow \mathbb{R}$ is a measurable 'cost function' such that $\varphi((1,0, \ldots))=0$.

We are interested in the total energy $\mathrm{E}^{(x)}(\eta)$ used to reduce an initial fragment of size $x$ to fragments that reach, for the first time, sizes of less than $\eta$; that is

$$
\mathrm{E}^{(\boldsymbol{x})}(\eta)=\sum_{t \geq 0} \mathbf{1}_{\left\{X_{k(t)}\left(t_{-}, \boldsymbol{x}\right) \geq \eta\right\}} X_{k(t)}^{\beta}\left(t_{-}, \boldsymbol{x}\right) \varphi(\Delta(t)) .
$$

We will simply write

$$
\mathrm{E}(\eta):=\mathrm{E}^{(1,0, \ldots)}(\eta)
$$

The following consequence of the homogeneity property will be useful.

Lemma 1. Let $\boldsymbol{x}=\left(x_{1}, x_{2}, \ldots\right) \in s^{\downarrow}$ and $\eta \in[0,1]$. We have

$$
\mathrm{E}^{(x)}(\eta) \stackrel{\text { LAW }}{=} \sum_{i} \mathbf{1}_{\left\{x_{i} \geq \eta\right\}} x_{i}^{\beta} \mathrm{E}_{i}\left(\frac{\eta}{x_{i}}\right)
$$

where, for each $i \geq 1, \mathrm{E}_{i}(\cdot)$ is the energy of a fragmentation process $\boldsymbol{X}^{(i)}$ issued from $(1,0, \ldots)$ with the same characteristics as $\boldsymbol{X}$, and the copies $\left(\boldsymbol{X}^{(i)}: i \geq 1\right)$ are independent.

Proof. Let $\left(\left(\Delta_{i}(t), k_{i}(t)\right): t \geq 0\right), i \geq 1$, be independent and identically distributed Poisson point processes with intensity measure $v \otimes \sharp$. Denote by $\overline{\boldsymbol{X}}^{\left(x_{i}\right)}, i \geq 1$, the sequence of independent homogeneous fragmentation processes constructed from the latter processes, respectively starting from $\left(x_{i}, 0, \ldots\right)$. From the branching property of $\boldsymbol{X}$ we have the identity

$$
\mathrm{E}^{(x)}(\eta) \stackrel{\text { LAW }}{=} \sum_{i} \sum_{t \geq 0} \mathbf{1}_{\left\{x_{i} \geq \eta\right\}} \mathbf{1}_{\left\{\bar{X}_{k_{i}(t)}^{\left(x_{i}\right)}\left(t_{-}\right) \geq \eta\right\}}\left(\bar{X}_{k_{i}(t)}^{\left(x_{i}\right)}\right)^{\beta}\left(t_{-}\right) \varphi\left(\Delta_{i}(t)\right) .
$$

Denoting now by $\left(\left(\Delta^{(i)}(t), k^{(i)}(t)\right): t \geq 0\right), i \geq 1$, the family of independent and identically distributed Poisson point processes associated with the process $\boldsymbol{X}^{(i)}$, we get, by homogeneity,

$$
\mathrm{E}^{(x)}(\eta) \stackrel{\text { LAW }}{=} \sum_{i} \sum_{t \geq 0} \mathbf{1}_{\left\{x_{i} \geq \eta\right\}} \mathbf{1}_{\left\{x_{i} X_{k^{(i)}(t)}^{(i)}\left(t_{-}\right) \geq \eta\right\}} x_{i}^{\beta}\left(X_{k^{(i)}(t)}^{(i)}\right)^{\beta}\left(t_{-}\right) \varphi\left(\Delta^{(i)}(t)\right),
$$

and the statement follows. 


\subsection{The energy of a two-step fragmentation procedure}

To formulate our problem, we introduce a second Poisson point process $\hat{\boldsymbol{K}}=((\hat{\Delta}(t), \hat{k}(t))$, $t \geq 0$ ) with values in $\delta^{\downarrow} \times \mathbb{N}$, and with intensity measure $\hat{v} \otimes \sharp$, where $\hat{v}$ is a dislocation measure satisfying the same assumptions as $v$. We can then simultaneously define a family of fragmentation processes $\hat{\boldsymbol{X}}=(\hat{X}(t, \boldsymbol{x}): t \geq 0)$ indexed by the initial condition $\boldsymbol{x}=\left(x_{1}, x_{2}, \ldots\right)$. We denote by $\hat{\alpha}$ the Malthusian exponent of $\hat{v}$. The energy used in the second fragmentation process is assumed to take the same form as for the first, in terms of (possibly different) parameters $\hat{\beta}$ and $\hat{\varphi}$.

We assume that $\boldsymbol{K}$ and $\hat{\boldsymbol{K}}$ are independent, so the families of fragmentation processes $\boldsymbol{X}$ and $\hat{\boldsymbol{X}}$ are independent, respectively called the first and second fragmentation processes.

In the sequel we assume that the first fragmentation process $\boldsymbol{X}$ is issued from the unitary fragment $(1,0, \ldots)$. Let $1 \geq \eta \geq \eta_{0}>0$. We let each mass fragment evolve in the first fragmentation process until the instant it first becomes smaller than $\eta$. Then it immediately enters the second fragmentation process $\hat{\boldsymbol{X}}$, and then evolves until it first becomes smaller than $\eta_{0}$.

For each $\eta \in(0,1]$, let $\boldsymbol{x}^{\eta} \in \mathcal{s}^{\downarrow}$ be the mass partition given by the 'output' of $\boldsymbol{X}$ when each of the fragments reaches for the first time a size less than $\eta$. More precisely, each fragment is 'frozen' at that time, while other (larger than $\eta$ ) fragments continue their independent evolutions. We write

$$
\boldsymbol{x}^{\eta}=\left(x_{1}^{\eta}, x_{2}^{\eta}, \ldots\right)
$$

for the decreasing rearrangement of the (random) frozen sizes of fragments when exiting the first fragmentation process. By the homogeneity and branching properties, if $\mathcal{E}\left(\eta, \eta_{0}\right)$ denotes the total energy spent in reducing the unit-size fragment by these procedures, we have the identity

$$
\mathcal{E}\left(\eta, \eta_{0}\right) \stackrel{\mathrm{LAW}}{=} \mathrm{E}(\eta)+\hat{\mathrm{E}}^{\left(x^{\eta}\right)}\left(\eta_{0}\right)
$$

where $\hat{E}^{(x)}(\cdot)$ is the energy of a copy of the second fragmentation process $\hat{\boldsymbol{X}}$ starting from $\boldsymbol{x}$, independent of the first fragmentation process.

Remark 1. Note that $\mathcal{E}\left(1, \eta_{0}\right)$ is the energy required to dislocate only the unit mass fragment with the first fragmentation process, and to then immediately use the second fragmentation process to break the resulting blocks, as long as their sizes are greater or equal to $\eta_{0}$ (the other fragments immediately exit the system). We will denote by $\mathscr{E}\left(1^{+}, \eta_{0}\right)=\hat{\mathrm{E}}\left(\eta_{0}\right)$ the total energy required when only the second fragmentation process is used from the beginning.

For the quantity $\mathscr{E}\left(\eta_{0}, \eta_{0}\right)=\mathrm{E}\left(\eta_{0}\right)$, no confusion arises: it corresponds to the case when the first fragmentation process is used during the whole procedure.

Our goal now is to compute the expectation of $\varepsilon\left(\eta, \eta_{0}\right)$.

The notation $\hat{\xi}, \hat{T}_{\eta}, \hat{\Pi}, \hat{U}$, and so on, will be used for the analogous objects associated with the fragmentation process $\hat{\boldsymbol{X}}$.

So far, the notation $\mathrm{P}$ has been used to denote the law of $\xi$. In the sequel, we keep the same notation $P$ to denote the product law of independent copies of the processes $\xi$ and $\hat{\xi}$ in the product path space. Extending the definition in (2) accordingly, we will also denote by $\tilde{\mathrm{P}}$ the product measure, the first marginal of which is given by $\left.\mathrm{d} \tilde{\mathrm{P}}\right|_{\mathcal{F}_{t}}=\left.\mathrm{e}^{(1-\alpha) \xi(t)} \mathrm{dP}\right|_{\mathcal{F}_{t}}$ and the second one given by $\left.\mathrm{d} \tilde{\mathrm{P}}\right|_{\hat{\mathcal{F}}_{t}}=\left.\mathrm{e}^{(1-\hat{\alpha}) \hat{\xi}(t)} \mathrm{dP}\right|_{\hat{\mathcal{F}}_{t}}$. Here $\left(\mathcal{F}_{t}: t \geq 0\right)$ and $\left(\hat{\mathcal{F}}_{t}: t \geq 0\right)$ are the natural filtrations of $\xi$ and $\hat{\xi}$, respectively.

We will assume throughout that the following integrability condition holds:

$$
\varphi \in L^{1}(v) \text { and } \hat{\varphi} \in L^{1}(\hat{v}) .
$$


In this case we define

$$
C=\int_{S} \varphi(s) \nu(\mathrm{d} s) \text { and } \hat{C}=\int_{S} \hat{\varphi}(s) \hat{v}(\mathrm{~d} s) .
$$

Let us introduce the functions

$$
\Psi(x)=C \int_{0}^{x} \mathrm{e}^{(\alpha-\beta) y} U(\mathrm{~d} y), \quad \hat{\Psi}(x)=\hat{C} \int_{0}^{x} \mathrm{e}^{(\hat{\alpha}-\hat{\beta}) y} \hat{U}(\mathrm{~d} y), \quad x \geq 0 .
$$

To simplify the notation, we will set, for all $a>0, \ell(a):=\log (1 / a)$.

We now have the elements necessary to compute the expected energy requirement in the two-step fragmentation procedure.

Lemma 2. Assume that the integrability condition (6) is satisfied. Let $\eta_{0} \in(0,1)$. Then, we have, for $\eta_{0}<\eta<1$,

$$
\begin{aligned}
\mathrm{E}\left(\mathcal{E}\left(\eta, \eta_{0}\right)\right)= & C \int_{0}^{\ell(\eta)} \mathrm{e}^{(\alpha-\beta) y} U(\mathrm{~d} y) \\
& +\hat{C} \int_{0}^{\ell(\eta)} \int_{\ell(\eta)-y}^{\ell\left(\eta_{0}\right)-y} \mathrm{e}^{(\alpha-\hat{\beta})(z+y)}\left[\int_{0}^{\ell\left(\eta_{0}\right)-(z+y)} \mathrm{e}^{(\hat{\alpha}-\hat{\beta}) x} \hat{U}(\mathrm{~d} x)\right] \Pi(\mathrm{d} z) U(\mathrm{~d} y) \\
= & \Psi(\ell(\eta))+\tilde{\mathrm{E}}\left(\mathbf{1}_{\left\{\xi_{T_{\eta}}<\ell\left(\eta_{0}\right)\right\}} \mathrm{e}^{(\alpha-\hat{\beta}) \xi_{T_{\eta}}} \hat{\Psi}\left(\ell\left(\eta_{0}\right)-\xi_{T_{\eta}}\right)\right)
\end{aligned}
$$

and

$$
\mathrm{E}\left(\mathcal{E}\left(\eta_{0}, \eta_{0}\right)\right)=\mathrm{E}\left(\mathrm{E}\left(\eta_{0}\right)\right)=\Psi\left(\ell\left(\eta_{0}\right)\right), \quad \mathrm{E}\left(\mathcal{E}\left(1^{+}, \eta_{0}\right)\right)=\mathrm{E}\left(\hat{\mathrm{E}}\left(\eta_{0}\right)\right)=\hat{\Psi}\left(\ell\left(\eta_{0}\right)\right) .
$$

When the renewal measure $U(\mathrm{~d} x)$ has no atom at 0 , we have

$$
\mathrm{E}\left(\mathcal{E}\left(1^{+}, \eta_{0}\right)\right)=\mathrm{E}\left(\mathcal{E}\left(1, \eta_{0}\right)\right)
$$

Proof. The proof is an extension of the arguments given in [6] corresponding to the case ' $\eta=1^{+}$' or $\eta=\eta_{0}$ and which we repeat here for convenience. By the compensation formula for the Poisson point process $(\Delta(u), k(u))$ associated with the first fragmentation process $\boldsymbol{X}$, we obtain, for $\eta_{0} \in(0,1]$,

$$
\begin{aligned}
\mathrm{E}\left(\mathrm{E}\left(\eta_{0}\right)\right) & =\mathrm{E}\left(\int_{0}^{\infty} \mathbf{1}_{\left\{\chi(t)>\eta_{0}\right\}}(\chi(t))^{\beta-1} \mathrm{~d} t\right) \int_{S} \varphi(s) v(\mathrm{~d} s) \\
& =C \mathrm{E}\left(\int_{0}^{\infty} \mathbf{1}_{\left\{\xi_{t}<\ell\left(\eta_{0}\right)\right\}} \mathrm{e}^{(1-\beta) \xi_{t}} \mathrm{~d} t\right)
\end{aligned}
$$

Thus,

$$
\mathrm{E}\left(\mathrm{E}\left(\eta_{0}\right)\right)=C \tilde{\mathrm{E}}\left(\int_{0}^{\infty} \mathbf{1}_{\left\{\xi_{t}<\ell\left(\eta_{0}\right)\right\}} \mathrm{e}^{(\alpha-\beta) \xi_{t}} \mathrm{~d} t\right)=C \int_{0}^{\ell\left(\eta_{0}\right)} \mathrm{e}^{(\alpha-\beta) y} U(\mathrm{~d} y)=\Psi\left(\ell\left(\eta_{0}\right)\right) .
$$

Similarly,

$$
\mathrm{E}\left(\hat{\mathrm{E}}\left(\eta_{0}\right)\right)=\hat{C} \int_{0}^{\ell\left(\eta_{0}\right)} \mathrm{e}^{(\hat{\alpha}-\hat{\beta}) y} \hat{U}(\mathrm{~d} y)=\hat{\Psi}\left(\ell\left(\eta_{0}\right)\right) .
$$

The above identity also implies that $\mathrm{E}\left(\mathscr{E}\left(1, \eta_{0}\right)\right)=\hat{C} \int_{0^{+}}^{\ell\left(\eta_{0}\right)} \mathrm{e}^{(\hat{\alpha}-\hat{\beta}) y} \hat{U}(\mathrm{~d} y)=\mathrm{E}\left(\hat{\mathrm{E}}\left(\eta_{0}\right)\right)$ when $U$ has no atom at 0 . 
The statement is thus proved for the cases ' $\eta=1^{+}$' and $\eta=\eta_{0}$. For the general case, we use Lemma 1 to obtain

$$
\begin{aligned}
\mathrm{E}\left(\hat{\mathrm{E}}^{\left(x^{\eta}\right)}\left(\eta_{0}\right)\right) & =\mathrm{E}\left(\sum_{i} \mathbf{1}_{\left\{x_{\eta, i}>\eta_{0}\right\}} x_{\eta, i}^{\hat{\beta}} \hat{\mathrm{E}}_{i}\left(\frac{\eta_{0}}{x_{\eta, i}}\right)\right) \\
& =\mathrm{E}\left(\sum_{i} \mathbf{1}_{\left\{x_{\eta, i}>\eta_{0}\right\}} x_{\eta, i}^{\hat{\beta}} \mathrm{E}\left(\hat{\mathrm{E}}_{i}\left(\frac{\eta_{0}}{x_{\eta, i}}\right) \mid x_{\eta, i}\right)\right) \\
& =\mathrm{E}\left(\left.\mathbf{1}_{\left\{\chi\left(T_{\eta}\right)>\eta_{0}\right\}}\left(\chi\left(T_{\eta}\right)\right)^{\hat{\beta}-1} \hat{\mathrm{E}}\left(\hat{\mathrm{E}}\left(\frac{\eta_{0}}{y}\right)\right)\right|_{y=\chi\left(T_{\eta}\right)}\right),
\end{aligned}
$$

where $\hat{E}(\cdot)$ is the energy of a copy of the second fragmentation process, starting from the unit mass, and which is independent of the first fragmentation process, and the $\hat{\mathrm{E}}_{i}(\cdot)$ are independent copies of $\hat{\mathrm{E}}(\cdot)$. Then, since $\chi(t)=\mathrm{e}^{-\xi_{t}}$, we have

$$
\begin{aligned}
\mathrm{E}\left(\hat{\mathrm{E}}^{\left(\boldsymbol{x}^{\eta}\right)}\left(\eta_{0}\right)\right) & =\tilde{\mathrm{E}}\left(\left.\mathbf{1}_{\left\{\xi_{T_{\eta}}<\ell\left(\eta_{0}\right)\right\}} \mathrm{e}^{(\alpha-\hat{\beta}) \xi_{T_{\eta}}} \tilde{\mathrm{E}}\left(\hat{\mathrm{E}}\left(\eta_{0} \mathrm{e}^{z}\right)\right)\right|_{z=\xi_{T_{\eta}}}\right) \\
& =\tilde{\mathrm{E}}\left(\mathbf{1}_{\left\{\xi_{T_{\eta}}<\ell\left(\eta_{0}\right)\right\}} \mathrm{e}^{(\alpha-\hat{\beta}) \xi_{T_{\eta}}} \hat{\Psi}\left(\ell\left(\eta_{0}\right)-\xi_{T_{\eta}}\right)\right)
\end{aligned}
$$

According to Lemma 1.10 of [3], the distribution of $\xi_{T_{\eta}}$ under $\tilde{\mathrm{P}}$ is given by

$$
\tilde{\mathrm{P}}\left(\xi_{T_{\eta}} \in \mathrm{d} z\right)=\int_{0}^{\ell(\eta)} \mathbf{1}_{\{\ell(\eta)<z\}} \Pi(\mathrm{d} z-y) U(\mathrm{~d} y) .
$$

Therefore,

$$
\mathrm{E}\left(\hat{\mathrm{E}}^{\left(x^{\eta}\right)}\left(\eta_{0}\right)\right)=\int_{0}^{\ell(\eta)}\left[\int_{\ell(\eta)-y}^{\ell\left(\eta_{0}\right)-y} \mathrm{e}^{(\alpha-\hat{\beta})(z+y)} \hat{\Psi}(\ell(\eta)-(z+y)) \Pi(\mathrm{d} z)\right] U(\mathrm{~d} y) .
$$

By bringing the pieces together and using identity (5), we obtain the result.

By analogy with (4), we introduce the notation

$$
\hat{\boldsymbol{x}}^{\eta}=\left(\hat{x}_{1}^{\eta}, \hat{x}_{2}^{\eta}, \ldots\right)
$$

for the decreasing rearrangement of the frozen sizes of fragments smaller than $\eta$ that exit the second fragmentation process started from the unit mass. The following decompositions of the total energy will be useful in the sequel.

Remark 2. For $1 \geq \eta \geq \eta_{0}>0$, we have

$$
\hat{\mathrm{E}}\left(\eta_{0}\right)=\hat{\mathrm{E}}(\eta)+\hat{\mathrm{E}}^{\left(\hat{x}^{\eta}\right)}\left(\eta_{0}\right)
$$

whence

$$
\mathcal{E}\left(\eta, \eta_{0}\right)-\mathcal{E}\left(1^{+}, \eta_{0}\right)=\mathrm{E}(\eta)-\hat{\mathrm{E}}(\eta)+\hat{\mathrm{E}}^{\left(x^{\eta}\right)}\left(\eta_{0}\right)-\hat{\mathrm{E}}^{\left(\hat{x}^{\eta}\right)}\left(\eta_{0}\right) .
$$

From this relation and by similar computations as in Lemma 2 , we can write

$$
\begin{aligned}
\mathrm{E}\left(\mathcal{E}\left(\eta, \eta_{0}\right)-\mathscr{E}\left(1^{+}, \eta_{0}\right)\right)= & \Psi(\ell(\eta))-\hat{\Psi}(\ell(\eta)) \\
& +\tilde{\mathrm{E}}\left(\mathbf{1}_{\left\{\xi_{T_{\eta}}<\ell\left(\eta_{0}\right)\right\}} \mathrm{e}^{(\alpha-\hat{\beta}) \xi T_{\eta}} \hat{\Psi}\left(\ell\left(\eta_{0}\right)-\xi_{T_{\eta}}\right)\right) \\
& -\tilde{\mathrm{E}}\left(\mathbf{1}_{\left\{\hat{\xi}_{\hat{T}_{\eta}}<\ell\left(\eta_{0}\right)\right\}} \mathrm{e}^{(\hat{\alpha}-\hat{\beta}) \hat{\xi}_{\hat{T}_{\eta}}} \hat{\Psi}\left(\ell\left(\eta_{0}\right)-\hat{\xi}_{\hat{T}_{\eta}}\right)\right) .
\end{aligned}
$$


Observe that when $U$ has no atom at 0 , we can replace $\mathcal{E}\left(1^{+}, \eta_{0}\right)$ by $\mathcal{E}\left(1, \eta_{0}\right)$ on the left-hand side of the formula.

Similarly, we have

$$
\begin{aligned}
\mathrm{E}(\mathcal{E}( & \left.\left.\eta, \eta_{0}\right)-\mathcal{E}\left(\eta_{0}, \eta_{0}\right)\right) \\
& =\mathrm{E}\left(\hat{\mathrm{E}}^{\left(x^{\eta}\right)}\left(\eta_{0}\right)-\mathrm{E}^{\left(x^{\eta}\right)}\left(\eta_{0}\right)\right) \\
& =\tilde{\mathrm{E}}\left(\mathbf{1}_{\left\{\xi_{T_{\eta}}<\ell\left(\eta_{0}\right)\right\}} \mathrm{e}^{(\alpha-\hat{\beta}) \xi_{T_{\eta}}} \hat{\Psi}\left(\ell\left(\eta_{0}\right)-\xi_{T_{\eta}}\right)-\mathbf{1}_{\left\{\xi_{T_{\eta}}<\ell\left(\eta_{0}\right)\right\}} \mathrm{e}^{(\alpha-\beta) \xi_{T_{\eta}}} \Psi\left(\ell\left(\eta_{0}\right)-\xi_{T_{\eta}}\right)\right)
\end{aligned}
$$

\section{Small thresholds}

In this section we consider the total energy $\mathrm{E}\left(\mathcal{E}\left(\eta, \eta_{0}\right)\right)$ when $\eta_{0}$ and $\eta$ go to 0 jointly in a suitable way. Our goal is to compare it with the mean energy required for reducing the unit fragment to fragments smaller than $\eta_{0}$ using only the first or second fragmentation process. We will assume that the quantities

$$
m(\alpha):=\int_{s \downarrow} \sum_{i=1}^{\infty} s_{n}^{\alpha} \log \left(\frac{1}{s_{n}^{\alpha}}\right) v(\mathrm{~d} \boldsymbol{s}) \quad \text { and } \quad \hat{m}(\hat{\alpha}):=\int_{s \downarrow} \sum_{i=1}^{\infty} s_{n}^{\hat{\alpha}} \log \left(\frac{1}{s_{n}^{\hat{\alpha}}}\right) \hat{v}(\mathrm{~d} s)
$$

are finite. Moreover, we impose the conditions

$$
\beta<\alpha \text { and } \hat{\beta}<\hat{\alpha} .
$$

These assumptions are physically reasonable, since the energies $\Psi(\infty)$ and $\hat{\Psi}(\infty)$ required to ensure that all the fragments vanish in the first and, respectively, second fragmentation processes are otherwise finite (see Remark 1 of [6]).

The following asymptotic result on the mean energy of a single fragmentation process is simply adapted from Lemma 4 of [6]; see also Theorem 1 therein.

Lemma 3. Under the previous assumptions, we have

$$
\lim _{\eta \rightarrow 0} \eta^{\alpha-\beta} \mathrm{E}(\mathrm{E}(\eta))=\frac{C}{(\alpha-\beta) m(\alpha)} \quad \text { and } \quad \lim _{\eta \rightarrow 0} \eta^{\hat{\alpha}-\hat{\beta}} \mathrm{E}(\hat{\mathrm{E}}(\eta))=\frac{\hat{C}}{(\hat{\alpha}-\hat{\beta}) \hat{m}(\hat{\alpha})} .
$$

The proof of Lemma 3 is based on the renewal theorem for subordinators (see [7]). From the latter theorem we also have, as $\eta \rightarrow 0^{+}$,

$$
\tilde{\mathrm{P}}\left(\xi_{T_{\eta}}-\ell(\eta) \in \mathrm{d} u\right) \rightarrow M(\mathrm{~d} u):=\frac{1}{m(\alpha)} \int_{\mathbb{R}^{+}} \Pi(y+\mathrm{d} u) \mathrm{d} y
$$

and

$$
\tilde{\mathrm{P}}\left(\hat{\xi}_{\hat{T}_{\eta}}-\ell(\eta) \in \mathrm{d} u\right) \rightarrow \hat{M}(\mathrm{~d} u):=\frac{1}{\hat{m}(\hat{\alpha})} \int_{\mathbb{R}^{+}} \hat{\Pi}(y+\mathrm{d} u) \mathrm{d} y,
$$

in the weak sense. Let us define, for $\lambda>0$ a fixed parameter, the finite and strictly positive constants

$$
\begin{aligned}
& F_{\lambda}:=\int_{0}^{\lambda} \mathrm{e}^{(\alpha-\hat{\beta}) u} \hat{\Psi}(\lambda-u) M(\mathrm{~d} u), \\
& D_{\lambda}:=\int_{0}^{\lambda} \mathrm{e}^{(\alpha-\beta) u} \Psi(\lambda-u) M(\mathrm{~d} u), \\
& \hat{D}_{\lambda}:=\int_{0}^{\lambda} \mathrm{e}^{(\hat{\alpha}-\hat{\beta}) u} \hat{\Psi}(\lambda-u) \hat{M}(\mathrm{~d} u) .
\end{aligned}
$$


We fix in the sequel the parameter $\lambda>0$. With these elements, we are in position to explicitly study the (comparative) behavior of the total energy for small thresholds $\eta$ and $\eta_{0}$, when these are bound by the relation $\eta_{0}=\eta \mathrm{e}^{-\lambda}$.

Theorem 1. (Two-step procedure versus first fragmentation only.) Assume that the renewal measure $U(\mathrm{~d} x)$ has no atom at 0 . For any $\lambda>0$, the following assertions hold.

(a) If $\hat{\beta}>\beta$ then, for all $\varepsilon \in\left(0, D_{\lambda}\right)$, there exists an $\eta_{\varepsilon} \in(0,1)$ such that, for all $\eta \leq \eta_{\varepsilon}$,

$$
\mathrm{E}\left(\varepsilon\left(\eta, \eta \mathrm{e}^{-\lambda}\right)\right)-\mathrm{E}\left(\mathrm{E}\left(\eta \mathrm{e}^{-\lambda}\right)\right) \leq\left(\varepsilon-D_{\lambda}\right) \eta^{\beta-\alpha}<0 .
$$

(b) If $\hat{\beta}<\beta$ then, for all $R>0$, there exists an $\eta_{R} \in(0,1)$ such that, for all $\eta \leq \eta_{R}$,

$$
\mathrm{E}\left(\mathcal{E}\left(\eta, \eta \mathrm{e}^{-\lambda}\right)\right)-\mathrm{E}\left(\mathrm{E}\left(\eta \mathrm{e}^{-\lambda}\right)\right) \geq R \eta^{\beta-\alpha}>0 .
$$

(c) If $\hat{\beta}=\beta$ then, for all $\varepsilon \in(0,1)$, there exists an $\eta_{\varepsilon} \in(0,1)$ such that, for all $\eta \leq \eta_{\varepsilon}$,

$$
\left(-\varepsilon+F_{\lambda}-D_{\lambda}\right) \eta^{\beta-\alpha} \leq \mathrm{E}\left(\varepsilon\left(\eta, \eta \mathrm{e}^{-\lambda}\right)\right)-\mathrm{E}\left(\mathrm{E}\left(\eta \mathrm{e}^{-\lambda}\right)\right) \leq\left(\varepsilon+F_{\lambda}-D_{\lambda}\right) \eta^{\beta-\alpha} .
$$

In all parts, we can replace $\eta^{\beta-\alpha}$ by $(\alpha-\beta) m(\alpha) \mathrm{E}(\mathrm{E}(\eta)) / C$.

Proof. From (9) in Remark 2 we obtain

$$
\begin{aligned}
& \eta^{\alpha-\beta}\left(\mathrm{E}\left(\mathcal{E}\left(\eta, \eta \mathrm{e}^{-\lambda}\right)\right)-\mathrm{E}\left(\mathcal{E}\left(\eta \mathrm{e}^{-\lambda}, \eta \mathrm{e}^{-\lambda}\right)\right)\right) \\
&=\tilde{\mathrm{E}}\left(\mathrm{e}^{(\alpha-\hat{\beta})\left(\xi_{T_{\eta}}-\ell(\eta)\right)} \hat{\Psi}\left(\lambda-\left(\xi_{T_{\eta}}-\ell(\eta)\right)\right) \mathbf{1}_{\left\{\xi_{T_{\eta}}-\ell(\eta)<\lambda\right\}}\right) \eta^{\hat{\beta}-\beta} \\
&-\tilde{\mathrm{E}}\left(\mathrm{e}^{(\alpha-\beta)\left(\xi_{T_{\eta}}-\ell(\eta)\right)} \Psi\left(\lambda-\left(\xi_{T_{\eta}}-\ell(\eta)\right)\right) \mathbf{1}_{\left\{\xi_{T_{\eta}}-\ell(\eta)<\lambda\right\}}\right) .
\end{aligned}
$$

By the weak convergence result (10) for $\tilde{\mathrm{P}}\left(\xi_{T_{\eta}}-\ell(\eta) \in \mathrm{d} y\right)$, the first and second expectations on the right-hand side of the above equation respectively converge to $F_{\lambda}$ and $D_{\lambda}$ (we use the fact that the limiting law is absolutely continuous). Then, we use the first identity in (7) to obtain the assertions of parts (a), (b), and (c), according to the relations between $\beta$ and $\hat{\beta}$. The last assertion follows from Lemma 3.

Theorem 2. (Two-step procedure versus second fragmentation only.) Assume that $U(\mathrm{~d} x)$ and $\hat{U}(\mathrm{~d} x)$ have no atom at 0 . For any $\lambda>0$, the following assertions hold.

(a) If $\hat{\alpha}<\alpha$, or if $\hat{\alpha}-\hat{\beta}<\alpha-\beta$, then, for all $R>0$, there exists an $\eta_{R} \in(0,1)$ such that, for all $\eta \leq \eta_{R}$

$$
\mathrm{E}\left(\mathcal{E}\left(\eta, \eta \mathrm{e}^{-\lambda}\right)\right)-\mathrm{E}\left(\hat{\mathrm{E}}\left(\eta \mathrm{e}^{-\lambda}\right)\right) \geq R \eta^{\hat{\beta}-\hat{\alpha}}>0 .
$$

(b) If $\hat{\alpha}>\alpha$ and $\hat{\alpha}-\hat{\beta}>\alpha-\beta$, then, for all $\varepsilon \in\left(0, \hat{D}_{\lambda}+\hat{C} /(\hat{\alpha}-\hat{\beta}) \hat{m}(\hat{\alpha})\right)$, there exists an $\eta_{\varepsilon} \in(0,1)$ such that, for all $\eta \leq \eta_{\varepsilon}$,

$$
\mathrm{E}\left(\mathscr{E}\left(\eta, \eta \mathrm{e}^{-\lambda}\right)\right)-\mathrm{E}\left(\hat{\mathrm{E}}\left(\eta \mathrm{e}^{-\lambda}\right)\right) \leq\left(\varepsilon-\hat{D}_{\lambda}-\frac{\hat{C}}{(\hat{\alpha}-\hat{\beta}) \hat{m}(\hat{\alpha})}\right) \eta^{\hat{\beta}-\hat{\alpha}}<0 .
$$

(c) If $\hat{\alpha}=\alpha$ and $\beta \geq \hat{\beta}$, then, for all $\varepsilon \in(0,1)$, there exists an $\eta_{\varepsilon}$ such that, for all $\eta \leq \eta_{\varepsilon}$,

$$
\begin{aligned}
\left(-\varepsilon+F_{\lambda}-\hat{D}_{\lambda}-\frac{\tilde{C}}{(\hat{\alpha}-\hat{\beta}) \hat{m}(\hat{\alpha})}\right) \eta^{\hat{\beta}-\hat{\alpha}} & \leq \mathrm{E}\left(\varepsilon\left(\eta, \eta \mathrm{e}^{-\lambda}\right)\right)-\mathrm{E}\left(\hat{\mathrm{E}}\left(\eta \mathrm{e}^{-\lambda}\right)\right) \\
& \leq\left(\varepsilon+F_{\lambda}-\hat{D}_{\lambda}-\frac{\tilde{C}}{(\hat{\alpha}-\hat{\beta}) \hat{m}(\hat{\alpha})}\right) \eta^{\hat{\beta}-\hat{\alpha}},
\end{aligned}
$$

where $\tilde{C}=\hat{C}$ if $\beta>\hat{\beta}$ and $\tilde{C}=\hat{C}-C$ if $\beta=\hat{\beta}$.

In all parts, we can replace $\eta^{\hat{\beta}-\hat{\alpha}}$ by $(\hat{\alpha}-\hat{\beta}) \hat{m}(\hat{\alpha}) \mathrm{E}(\hat{\mathrm{E}}(\eta)) / \hat{C}$. 
Proof. From (8) in Remark 2 we obtain

$$
\begin{aligned}
\eta^{\hat{\alpha}-\hat{\beta}}\left(\mathrm{E}\left(\mathscr{E}\left(\eta, \eta \mathrm{e}^{-\lambda}\right)\right)-\mathrm{E}\left(\mathcal{E}\left(1, \eta \mathrm{e}^{-\lambda}\right)\right)\right) \\
=\tilde{\mathrm{E}}\left(\mathrm{e}^{(\alpha-\hat{\beta})\left(\xi_{T_{\eta}}-\ell(\eta)\right)} \hat{\Psi}\left(\lambda-\left(\xi_{T_{\eta}}-\ell(\eta)\right)\right) \mathbf{1}_{\left\{\xi_{T_{\eta}}-\ell(\eta)<\lambda\right\}}\right) \eta^{\hat{\alpha}-\alpha} \\
\quad-\tilde{\mathrm{E}}\left(\mathrm{e}^{(\hat{\alpha}-\hat{\beta})\left(\hat{\xi}_{\hat{T}_{\eta}}-\ell(\eta)\right)} \hat{\Psi}\left(\lambda-\left(\hat{\xi}_{\hat{T}_{\eta}}-\ell(\eta)\right)\right) \mathbf{1}_{\left\{\hat{\xi}_{\hat{T}_{\eta}}-\ell(\eta)<\lambda\right\}}\right) \\
\quad+\eta^{\hat{\alpha}-\hat{\beta}} \Psi(\ell(\eta))-\eta^{\hat{\alpha}-\hat{\beta}} \hat{\Psi}(\ell(\eta)) .
\end{aligned}
$$

By the weak convergence result (11) for $\tilde{\mathrm{P}}\left(\hat{\xi}_{\hat{T}_{\eta}}-\ell(\eta) \in \mathrm{d} y\right)$, and using Lemma 3 and the identities in (7), the above expression is seen to converge as $\eta \rightarrow 0$ to

$$
\lim _{\eta \rightarrow 0} \eta^{\hat{\alpha}-\alpha} F_{\lambda}-\hat{D}_{\lambda}+\eta^{\hat{\alpha}-\hat{\beta}-(\alpha-\beta)} \frac{C}{(\alpha-\beta) m(\alpha)}-\frac{\hat{C}}{(\hat{\alpha}-\hat{\beta}) \hat{m}(\hat{\alpha})}
$$

(this limit exists in $\mathbb{R} \cup\{+\infty\}$ ). Hence, we obtain each of the three parts using the relations in (7) again. The last assertion follows from Lemma 3.

We next summarize the main results of this section in an asymptotic comparative scheme. The notation $\mathrm{Fr}_{1,2}$ refers to the situation where both devices are effectively used in the two-step fragmentation procedure (i.e. $\eta_{0} / \eta \in(0,1)$ ), whereas the notation $\mathrm{Fr}_{1}$ and $\mathrm{Fr}_{2}$ respectively refer to the situations where the first or second fragmentation process is used.

In the statement of the next result we use the following convention. We will say that the procedure $\operatorname{Fr}_{1,2}$ is better than $\mathrm{Fr}_{1}$ if, for each $\lambda>0, \mathrm{E}\left(\mathcal{E}\left(\eta, \eta \mathrm{e}^{-\lambda}\right)\right)<\mathrm{E}\left(\mathrm{E}\left(\eta \mathrm{e}^{-\lambda}\right)\right)$ as soon as $\eta$ is sufficiently small. Analogously, $\operatorname{Fr}_{2}$ is better than $\operatorname{Fr}_{1}$ if, for each $\lambda>0$, $\mathrm{E}\left(\hat{\mathrm{E}}\left(\eta \mathrm{e}^{-\lambda}\right)\right)<\mathrm{E}\left(\mathrm{E}\left(\eta \mathrm{e}^{-\lambda}\right)\right)$ as soon as $\eta$ is sufficiently small. The other comparisons are understood in a similar way.

Corollary 1. Assume that $U(\mathrm{~d} x)$ and $\hat{U}(\mathrm{~d} x)$ have no atom at 0 . Then,

$\hat{\alpha}>\alpha, \hat{\beta}<\beta$ (thus $\alpha-\beta<\hat{\alpha}-\hat{\beta}): F r_{1}$ is better than $F_{1,2}$, which is better than $F r_{2}$,

$\hat{\alpha}<\alpha, \hat{\beta}>\beta$ (thus $\alpha-\beta>\hat{\alpha}-\hat{\beta}$ ): Fr $r_{2}$ is better than $F_{1,2}$, which is better than Fr $r_{1}$,

$\hat{\alpha}<\alpha, \hat{\beta}<\beta$, and $\alpha-\beta<\hat{\alpha}-\hat{\beta}: F r_{1}$ is better than $F r_{2}$, which is better than $F r_{1,2}$,

$\hat{\alpha}<\alpha, \hat{\beta}<\beta$, and $\alpha-\beta>\hat{\alpha}-\hat{\beta}: F r_{2}$ is better than $F r_{1}$, which is better than $F r_{1,2}$,

$\hat{\alpha}>\alpha, \hat{\beta}>\beta$, and $\alpha-\beta<\hat{\alpha}-\hat{\beta}: F r_{1,2}$ is better than $F r_{1}$, which is better than $F r_{2}$,

$\hat{\alpha}>\alpha, \hat{\beta}>\beta$, and $\alpha-\beta>\hat{\alpha}-\hat{\beta}: F r_{2}$, is better than $F r_{1,2}$, which is better than $F r_{1}$.

Remark 3. By parts (c) of Theorems 1 and 2, if $\hat{\alpha}=\alpha$ or if $\hat{\beta}=\beta$, the comparative efficiencies of $\mathrm{Fr}_{1}, \mathrm{Fr}_{2}$, and $\mathrm{Fr}_{1,2}$ for small enough $\eta$ are in general determined by those parameters and by the value of $\lambda>0$.

\section{Close to unit size thresholds}

We now investigate the behavior of $\mathrm{E}\left(\mathcal{E}\left(\eta, \eta_{0}\right)\right)$ for large values of $\eta$ and $\eta_{0}$. Again, we will compare the mean energy of the two-step fragmentation procedure for the case in which only the second or first fragmentation process is used. 
We will assume in this analysis that the subordinators $\xi$ and $\hat{\xi}$ satisfy, under $\tilde{\mathrm{P}}$, a condition of regular variation at $\infty$. Namely, respectively denoting by $\tilde{\phi}$ and $\tilde{\hat{\phi}}$ their Laplace exponents (see (3)), we assume that

(RV) there exists $\rho, \hat{\rho} \in(0,1)$ such that, for all $\lambda \geq 0$,

$$
\lim _{q \rightarrow \infty} \frac{\tilde{\phi}(\lambda q)}{\tilde{\phi}(q)}=\lambda^{\rho}, \quad \lim _{q \rightarrow \infty} \frac{\tilde{\hat{\phi}}(\lambda q)}{\tilde{\hat{\phi}}(q)}=\lambda^{\hat{\rho}} .
$$

This assumption can be equivalently (and transparently) stated in terms of the infinitesimal behavior near $\eta=1$ of the 'mean energy functions' $\eta \mapsto \mathrm{E}(\mathrm{E}(\eta))$ and $\eta \mapsto \mathrm{E}(\hat{\mathrm{E}}(\eta))$ of each of the fragmentation processes. See Remark 5, below.

Recall that a function $G: \mathbb{R}_{+} \rightarrow \mathbb{R}_{+}$is said to vary slowly at 0 if $\lim _{x \rightarrow 0^{+}} G(\lambda x) / G(x)=1$ for all $\lambda \in[0, \infty)$. A well-known fact that will be used in the sequel is that such convergence is uniform in $\lambda \in\left[0, \lambda_{0}\right]$ for all $\lambda_{0} \in(0, \infty)$.

By $L$ and $\hat{L}$ we will denote the nonnegative slowly varying functions at 0 defined by the relations

$$
L\left(\frac{1}{x}\right)=\frac{1}{\Gamma(1+\rho)} \frac{x^{\rho}}{\tilde{\phi}(x)}, \quad \hat{L}\left(\frac{1}{x}\right)=\frac{1}{\Gamma(1+\hat{\rho})} \frac{x^{\hat{\rho}}}{\tilde{\hat{\phi}}(x)} .
$$

(Note that these definitions of the slowly varying functions $L$ and $\hat{L}$ correspond to the usual ones given in Section 0.7 of [2] or Theorem 5.13 of [9], but divided by the appropriate constants).

Remark 4. Using the aforementioned uniform convergence result for $L$ and $\hat{L}$, from relation (3), it is easy to check that

$$
\lim _{q \rightarrow \infty} \frac{\phi(q)}{\tilde{\phi}(q)}=\lim _{q \rightarrow \infty} \frac{\hat{\phi}(q)}{\tilde{\hat{\phi}}(q)}=1 .
$$

Consequently, assumption (RV) implies that the same condition holds on $\phi$ and $\hat{\phi}$, and vice versa. For a discussion of assumption (RV), see [9, p. 132].

We define

$$
Q_{\phi, \hat{\phi}}:=\lim _{q \rightarrow \infty} \frac{\hat{\phi}(q)}{\phi(q)}=\lim _{q \rightarrow \infty} \frac{\tilde{\hat{\phi}}(q)}{\tilde{\phi}(q)}=\lim _{x \rightarrow 0^{+}} \frac{L(x) x^{\rho}}{\hat{L}(x) x^{\hat{\rho}}} \frac{\Gamma(1+\rho)}{\Gamma(1+\hat{\rho})}
$$

if the limit in $[0, \infty]$ exists. More generally, we write

$$
Q_{\phi, \hat{\phi}}^{+}:=\limsup _{q \rightarrow \infty} \frac{\hat{\phi}(q)}{\phi(q)}=\limsup _{x \rightarrow 0^{+}} \frac{L(x) x^{\rho}}{\hat{L}(x) x^{\hat{\rho}}} \frac{\Gamma(1+\rho)}{\Gamma(1+\hat{\rho})}
$$

and

$$
Q_{\phi, \hat{\phi}}^{-}:=\liminf _{q \rightarrow \infty} \frac{\hat{\phi}(q)}{\phi(q)}=\liminf _{x \rightarrow 0^{+}} \frac{L(x) x^{\rho}}{\hat{L}(x) x^{\hat{\rho}}} \frac{\Gamma(1+\rho)}{\Gamma(1+\hat{\rho})} .
$$

Recall the notation

$$
\Psi(x)=C \int_{0}^{x} \mathrm{e}^{(\alpha-\beta) y} U(\mathrm{~d} y), \quad \hat{\Psi}(x)=\hat{C} \int_{0}^{x} \mathrm{e}^{(\hat{\alpha}-\hat{\beta}) y} \hat{U}(\mathrm{~d} y) .
$$


Lemma 4. We have

$$
\begin{aligned}
C Q_{\phi, \hat{\phi}}^{-} \frac{\Gamma(1+\hat{\rho})}{\Gamma(1+\rho)}-\hat{C} & \leq \liminf _{\eta \rightarrow 1^{-}} \frac{\Psi(\ell(\eta))-\hat{\Psi}(\ell(\eta))}{\hat{L}(\ell(\eta)) \ell(\eta)^{\hat{\rho}}} \\
& \leq \limsup _{\eta \rightarrow 1^{-}} \frac{\Psi(\ell(\eta))-\hat{\Psi}(\ell(\eta))}{\hat{L}(\ell(\eta)) \ell(\eta)^{\hat{\rho}}} \\
& \leq C Q_{\phi, \hat{\phi}}^{+} \frac{\Gamma(1+\hat{\rho})}{\Gamma(1+\rho)}-\hat{C}
\end{aligned}
$$

In particular,

$$
\begin{aligned}
& \lim _{\eta \rightarrow 1^{-}} \frac{\Psi(\ell(\eta))-\hat{\Psi}(\ell(\eta))}{\hat{L}(\ell(\eta)) \ell(\eta)^{\hat{\rho}}} \\
& \quad= \begin{cases}\infty & \text { if } \hat{\rho}>\rho, \\
-\hat{C} & \text { if } \hat{\rho}<\rho, \\
C Q_{\phi, \hat{\phi}}-\hat{C} & \text { if } \hat{\rho}=\rho \text { and there exists } Q_{\phi, \hat{\phi}}=\lim _{x \rightarrow 0^{+}} L(x) / \hat{L}(x) \in[0, \infty] .\end{cases}
\end{aligned}
$$

Proof. From the relation $\int_{0}^{\infty} \mathrm{e}^{-q x} U(\mathrm{~d} x)=1 / \tilde{\phi}(q)$ (see [5, Section III.1]) and by the Tauberian theorem (see [2, Section 0.7] or [9, Theorem 5.13]), our assumptions on $\tilde{\phi}$ and $\tilde{\hat{\phi}}$ are respectively equivalent to

$$
\lim _{x \rightarrow 0^{+}} \frac{U(x)}{x^{\rho} L(x)}=1, \quad \lim _{x \rightarrow 0^{+}} \frac{\hat{U}(x)}{x^{\hat{\rho}} \hat{L}(x)}=1 .
$$

On the other hand, we have

$$
\begin{aligned}
\Psi(x)-\hat{\Psi}(x) & \leq C \mathrm{e}^{|\alpha-\beta| x} U(x)-\hat{C} \mathrm{e}^{-|\hat{\alpha}-\hat{\beta}| x} \hat{U}(x) \\
& =\hat{L}(x) x^{\hat{\rho}} \hat{C}\left(\frac{C}{\hat{C}} \mathrm{e}^{|\alpha-\beta| x} \frac{U(x)}{x^{\rho} L(x)} \frac{L(x)}{\hat{L}(x)} x^{\rho-\hat{\rho}}-\mathrm{e}^{-|\hat{\alpha}-\hat{\beta}| x} \frac{\hat{U}(x)}{x^{\hat{\rho}} \hat{L}(x)}\right)
\end{aligned}
$$

and, similarly,

$$
\begin{aligned}
\Psi(x)-\hat{\Psi}(x) & \geq C \mathrm{e}^{-|\alpha-\beta| x} U(x)-\hat{C} \mathrm{e}^{|\hat{\alpha}-\hat{\beta}| x} \hat{U}(x) \\
& =\hat{L}(x) x^{\hat{\rho}} \hat{C}\left(\frac{C}{\hat{C}} \mathrm{e}^{-|\alpha-\beta| x} \frac{U(x)}{x^{\rho} L(x)} \frac{L(x)}{\hat{L}(x)} x^{\rho-\hat{\rho}}-\mathrm{e}^{|\hat{\alpha}-\hat{\beta}| x} \frac{\hat{U}(x)}{x^{\hat{\rho}} \hat{L}(x)}\right) .
\end{aligned}
$$

The first statement follows from these bounds. To complete the proof, note that, since $L(x) /$ $\hat{L}(x)$ is slowly varying at 0 , we have

$$
\lim _{x \rightarrow 0^{+}} \frac{L(x)}{\hat{L}(x)} x^{\rho-\hat{\rho}}= \begin{cases}\infty & \text { if } \hat{\rho}>\rho, \\ 0 & \text { if } \hat{\rho}<\rho, \\ Q_{\phi, \hat{\phi}} & \text { if } \hat{\rho}=\rho \text { and there exists } \lim _{x \rightarrow 0^{+}} L(x) / \hat{L}(x) \in[0, \infty],\end{cases}
$$

also using the fact that $\lim _{x \rightarrow 0^{+}} G(x)=0$ for any regularly varying (at 0 ) function $G(x)$ with positive index. 
Note that assumption (RV) implies that $U$ has no atom at 0 (see, e.g. the first lines of the previous proof).

Remark 5. The estimates used in the proof of Lemma 4 show that

$$
\Psi(x) \sim C U(x) \text { and } \hat{\Psi}(x) \sim \hat{C} \hat{U}(x) \text { when } x \rightarrow 0^{+},
$$

so that $\Psi(x) \sim C x^{\rho} L(x)$ and $\hat{\Psi}(x) \sim \hat{C} x^{\hat{\rho}} \hat{L}(x)$ as well. Consequently, from the equalities in (7), assumption (RV) is equivalent to

(RV) $x \mapsto \mathrm{E}\left(\mathrm{E}\left(\mathrm{e}^{-x}\right)\right)$ and $x \mapsto \mathrm{E}\left(\hat{\mathrm{E}}\left(\mathrm{e}^{-x}\right)\right)$ are regularly varying at $0^{+}$with indices $\rho, \hat{\rho} \in$ $(0,1)$, respectively.

This alternative formulation has the advantage of providing a way to infer the regularity indices from separate observations of both fragmentation processes, if we were able to measure the energies required to obtain fragments of different close to unit sizes. More precisely,

$$
\frac{\log \mathrm{E}\left(\mathrm{E}\left(\eta^{\lambda}\right)\right)-\log \mathrm{E}(\mathrm{E}(\eta))}{\log \lambda}
$$

should be close to $\rho$ for $\eta$ sufficiently close to 1 . Alternatively, $\rho$ could in principle also be deduced from the estimation method of $\phi$ developed in [8].

In the same vein, we remark that the existence of the limit $Q_{\phi, \hat{\phi}}$ is equivalent to

$$
\text { there exists } Q:=\lim _{\eta \rightarrow 1^{-}} \frac{\mathrm{E}(\mathrm{E}(\eta))}{\mathrm{E}(\hat{\mathrm{E}}(\eta))}=\lim _{\eta \rightarrow 1^{-}} \frac{C}{\hat{C}} Q_{\phi, \hat{\phi}} \frac{\Gamma(1+\hat{\rho})}{\Gamma(1+\rho)} .
$$

In general, Lemma 4 indeed shows that

$$
\hat{C}\left(Q^{-}-1\right) \leq \liminf _{\eta \rightarrow 1^{-}} \frac{\Psi(\ell(\eta))-\hat{\Psi}(\ell(\eta))}{\hat{L}(\ell(\eta)) \ell(\eta)^{\hat{\rho}}} \leq \limsup _{\eta \rightarrow 1^{-}} \frac{\Psi(\ell(\eta))-\hat{\Psi}(\ell(\eta))}{\hat{L}(\ell(\eta)) \ell(\eta)^{\hat{\rho}}} \leq \hat{C}\left(Q^{+}-1\right),
$$

where

$$
Q^{+}:=\limsup _{\eta \rightarrow 1^{-}} \frac{\mathrm{E}(\mathrm{E}(\eta))}{\mathrm{E}(\hat{\mathrm{E}}(\eta))}=\frac{C}{\hat{C}} Q_{\phi, \hat{\phi}}^{+} \frac{\Gamma(1+\hat{\rho})}{\Gamma(1+\rho)}
$$

and

$$
Q^{-}:=\liminf _{\eta \rightarrow 1^{-}} \frac{\mathrm{E}(\mathrm{E}(\eta))}{\mathrm{E}(\hat{\mathrm{E}}(\eta))}=\frac{C}{\hat{C}} Q_{\phi, \hat{\phi}}^{-} \frac{\Gamma(1+\hat{\rho})}{\Gamma(1+\rho)} .
$$

We now recall that, under our assumptions on the Laplace exponents $\tilde{\phi}$ and $\tilde{\hat{\phi}}$, by the DynkinLamperti theorem (see, for instance, Theorem 5.16 of [9]), it weakly holds that, as $\eta \rightarrow 1^{-}$,

$$
\tilde{\mathrm{P}}\left(\frac{\xi_{T_{\eta}}-\ell(\eta)}{\ell(\eta)} \in \mathrm{d} y\right) \rightarrow \mu(\mathrm{d} y):=\frac{\sin (\rho \pi)}{\pi} \frac{\mathrm{d} y}{(1+y) y^{\rho}}
$$

and

$$
\tilde{\mathrm{P}}\left(\frac{\hat{\xi}_{\hat{T}_{\eta}}-\ell(\eta)}{\ell(\eta)} \in \mathrm{d} y\right) \rightarrow \hat{\mu}(\mathrm{d} y):=\frac{\sin (\hat{\rho} \pi)}{\pi} \frac{\mathrm{d} y}{(1+y) y^{\hat{\rho}}} .
$$

This suggests the way in which $\eta$ and $\eta_{0}$ should go to 1 in order to observe a coherent close to unit size asymptotic behavior. In the sequel, $\gamma>1$ is a fixed parameter, and we assume that

$$
\eta_{0}=\eta_{0}(\eta)=\eta^{\gamma}
$$

We have the following result. 
Lemma 5. We have

$$
\begin{aligned}
& \lim _{\eta \rightarrow 1^{-}} \frac{\tilde{\mathrm{E}}\left(\mathbf{1}_{\left\{\xi_{T_{\eta}}<\ell\left(\eta_{0}\right)\right\}} \mathrm{e}^{(\alpha-\hat{\beta}) \xi_{T_{\eta}}} \hat{\Psi}\left(\ell\left(\eta_{0}\right)-\xi_{T_{\eta}}\right)\right)-\tilde{\mathrm{E}}\left(\mathbf{1}_{\left\{\hat{\xi}_{\hat{T}_{\eta}}<\ell\left(\eta_{0}\right)\right\}} \mathrm{e}^{\left.(\hat{\alpha}-\hat{\beta}) \hat{\xi}_{\hat{T}_{\eta}} \hat{\Psi}\left(\ell\left(\eta_{0}\right)-\hat{\xi}_{\hat{T}_{\eta}}\right)\right)}\right.}{(\ell(\eta) \hat{\rho} \hat{\rho} \hat{L}(\ell(\eta))} \\
& \quad=\hat{C}\left[\int_{0}^{\gamma-1}(\gamma-1-y)^{\hat{\rho}} \mu(\mathrm{d} y)-\int_{0}^{\gamma-1}(\gamma-1-y)^{\hat{\rho}} \hat{\mu}(\mathrm{d} y)\right] .
\end{aligned}
$$

Moreover, in the case $\frac{1}{2} \geq \rho>\hat{\rho}$, the limit is a nonnegative and increasing function of $\gamma$ for $\gamma \in[1,2]$, which goes to 0 when $\gamma \rightarrow 1^{+}$.

Proof. Denote by $\partial(\eta)$ the numerator on the left-hand side of (15), and denote respectively by $\mu^{\eta}$ and $\hat{\mu}^{\eta}$ the laws of

$$
\frac{\xi_{T_{\eta}}-\ell(\eta)}{\ell(\eta)} \text { and } \frac{\hat{\xi}_{T_{\eta}}-\ell(\eta)}{\ell(\eta)}
$$

We then easily see that

$$
\begin{aligned}
\partial(\eta) \leq & \mathrm{e}^{\gamma \ell(\eta)|\alpha-\hat{\beta}|} \int_{0}^{\gamma-1} \hat{\Psi}(\ell(\eta)(\gamma-1-y)) \mu^{\eta}(\mathrm{d} y) \\
& -\mathrm{e}^{-\gamma \ell(\eta)|\hat{\alpha}-\hat{\beta}|} \int_{0}^{\gamma-1} \hat{\Psi}(\ell(\eta)(\gamma-1-y)) \hat{\mu}^{\eta}(\mathrm{d} y)
\end{aligned}
$$

and

$$
\begin{aligned}
\partial(\eta) \geq & \mathrm{e}^{-\gamma \ell(\eta)|\alpha-\hat{\beta}|} \int_{0}^{\gamma-1} \hat{\Psi}(\ell(\eta)(\gamma-1-y)) \mu^{\eta}(\mathrm{d} y) \\
& -\mathrm{e}^{\gamma \ell(\eta)|\hat{\alpha}-\hat{\beta}|} \int_{0}^{\gamma-1} \hat{\Psi}(\ell(\eta)(\gamma-1-y)) \hat{\mu}^{\eta}(\mathrm{d} y) .
\end{aligned}
$$

On the other hand, by Remark 5 we have

$$
\theta(x):=\frac{\hat{\Psi}(x)}{\hat{C} \hat{L}(x) x^{\hat{\rho}}} \rightarrow 1
$$

when $x \searrow 0$, and, thus, $\theta(x)$ is slowly varying at 0 . Now fix $\varepsilon \in(0,1)$, and recall that, for a slowly varying at 0 function $G(x)$, the convergence $G(\lambda x) / G(x) \rightarrow 1$ is uniform in $\lambda \in\left[0, \lambda_{0}\right]$ for all $\lambda_{0} \in(0,1)$. Therefore, since

$$
\hat{\Psi}(\ell(\eta) y)=\frac{\theta(\ell(\eta) y)}{\theta(\ell(\eta))} \frac{\hat{L}(\ell(\eta) y)}{\hat{L}(\ell(\eta))} \hat{\Psi}(\ell(\eta)) y^{\hat{\rho}},
$$

and $\hat{L}$ varies slowly at 0 , we deduce that, if $\eta \in(0,1)$ is sufficiently close to 1 then, for all $y \in[0, \gamma-1]$,

$$
(1-\varepsilon) \hat{\Psi}(\ell(\eta)) y^{\hat{\rho}} \leq \hat{\Psi}(\ell(\eta) y) \leq(1+\varepsilon) \hat{\Psi}(\ell(\eta)) y^{\hat{\rho}} .
$$

Moreover, from (16), it follows that, if $\eta$ is sufficiently close to 1 then, for all $y \in[0, \gamma-1]$,

$$
\hat{C}(1-\varepsilon)^{2} y^{\hat{\rho}} \leq \frac{\hat{\Psi}(\ell(\eta) y)}{(\ell(\eta))^{\hat{\rho}} \hat{L}(\ell(\eta))} \leq \hat{C}(1+\varepsilon)^{2} y^{\hat{\rho}} .
$$


It follows that

$$
\limsup _{\eta \rightarrow 1^{-}} \frac{\partial(\eta)}{(\ell(\eta))^{\hat{\rho}} \hat{L}(\ell(\eta))} \leq(1+\varepsilon)^{2} \hat{C} A_{\gamma}-(1-\varepsilon)^{2} \hat{C} \hat{A}_{\gamma}
$$

and

$$
\liminf _{\eta \rightarrow 1^{-}} \frac{\partial(\eta)}{(\ell(\eta))^{\hat{\rho}} \hat{L}(\ell(\eta))} \geq(1-\varepsilon)^{2} \hat{C} A_{\gamma}-(1+\varepsilon)^{2} \hat{C} \hat{A}_{\gamma}
$$

where

$$
\begin{aligned}
& A_{\gamma}=\frac{\sin (\pi \rho)}{\pi} \int_{0}^{\gamma-1}(\gamma-1-u)^{\hat{\rho}} \frac{\mathrm{d} u}{(1+u) u^{\rho}}, \\
& \hat{A}_{\gamma}=\frac{\sin (\pi \hat{\rho})}{\pi} \int_{0}^{\gamma-1}(\gamma-1-u)^{\hat{\rho}} \frac{\mathrm{d} u}{(1+u) u^{\hat{\rho}}} .
\end{aligned}
$$

The first statement follows by letting $\varepsilon \rightarrow 0^{+}$. The asserted properties of $\hat{C}\left(A_{\gamma}-\hat{A}_{\gamma}\right)$ are a consequence of the inequalities $u^{-\rho}>u^{-\hat{\rho}}$ for $u \in(0,1)$ and $\sin (\pi \rho)>\sin (\pi \hat{\rho})>0$ when $\frac{1}{2}>\rho>\hat{\rho}$, and the dominated convergence.

Next we introduce helpful concepts in order to state our results on the energy for large thresholds. We use the definitions of $Q, Q^{+}$, and $Q^{-}$in (12), (13), and (14).

Definition 1. We say that the fragmentation process $\boldsymbol{X}$ is infinitesimally efficient (inf. eff.) compared to $\hat{\boldsymbol{X}}$ if (RV) holds and

$$
Q^{+}=\limsup _{\eta \rightarrow 1^{-}} \frac{\mathrm{E}(\mathrm{E}(\eta))}{\mathrm{E}(\hat{\mathrm{E}}(\eta))}<1
$$

Conversely, we say that the fragmentation process $\hat{\boldsymbol{X}}$ is inf. eff. compared to $\boldsymbol{X}$ if (RV) holds and

$$
Q^{-}=\liminf _{\eta \rightarrow 1^{-}} \frac{\mathrm{E}(\mathrm{E}(\eta))}{\mathrm{E}(\hat{\mathrm{E}}(\eta))}>1
$$

With our previous notation, this can be written as follows. The fragmentation process $\boldsymbol{X}$ is infinitesimally efficient compared to $\hat{\boldsymbol{X}}$ if $(\mathrm{RV})$ holds and

$$
Q_{\phi, \hat{\phi}}^{+}<\frac{\hat{C}}{C} \frac{\Gamma(1+\rho)}{\Gamma(1+\hat{\rho})} .
$$

Conversely, the fragmentation process $\hat{\boldsymbol{X}}$ is infinitesimally efficient compared to $\boldsymbol{X}$ if (RV) holds and

$$
Q_{\phi, \hat{\phi}}^{-}>\frac{\hat{C}}{C} \frac{\Gamma(1+\rho)}{\Gamma(1+\hat{\rho})} .
$$

For instance, $\boldsymbol{X}$ is infinitesimally efficient compared to $\hat{\boldsymbol{X}}$ if $\rho>\hat{\rho}$ or if $\rho=\hat{\rho}$ and $Q_{\phi, \hat{\phi}}$ exists in $[0, \hat{C} \Gamma(1+\rho) / C \Gamma(1+\hat{\rho}))$. Similarly, $\hat{\boldsymbol{X}}$ is infinitesimally efficient compared to $\hat{\boldsymbol{X}}$, e.g. if $\rho<\hat{\rho}$ or if $\rho=\hat{\rho}$ and $Q_{\phi, \hat{\phi}}$ exists in $(\hat{C} \Gamma(1+\rho) / C \Gamma(1+\hat{\rho}), \infty]$.

The following result is satisfied. 
Theorem 3. (Two-step procedure versus second fragmentation only.) For each $\gamma \in(1, \infty)$, the following assertions hold.

(a) If $\hat{\boldsymbol{X}}$ is infinitesimally efficient compared to $\boldsymbol{X}$ and $Q^{-}=Q=\infty$ (in particular, if $\hat{\rho}>\rho)$, then, for all $R>0$, there exists an $\eta_{R} \in(0,1)$ such that, for all $\eta \in\left(\eta_{R}, 1\right]$,

$$
\mathrm{E}\left(\mathcal{E}\left(\eta, \eta^{\gamma}\right)\right)-\mathrm{E}\left(\hat{\mathrm{E}}\left(\eta^{\gamma}\right)\right)>R\left[\tilde{\hat{\phi}}\left(\frac{1}{\ell(\eta)}\right)\right]^{-1}>0 .
$$

(b) If $\hat{\boldsymbol{X}}$ is infinitesimally efficient compared to $\boldsymbol{X}$ and $Q^{-} \in(1, \infty)$ (and, thus, $\left.\rho=\hat{\rho}\right)$, then, for all $\varepsilon \in\left(0, Q^{-}-1\right)$, there exists an $\eta_{\varepsilon} \in(0,1)$ such that, for all $\eta \in\left(\eta_{\varepsilon}, 1\right]$,

$$
\mathrm{E}\left(\varepsilon\left(\eta, \eta^{\gamma}\right)\right)-\mathrm{E}\left(\hat{\mathrm{E}}\left(\eta^{\gamma}\right)\right)>\left(Q^{-}-1-\varepsilon\right) \hat{C}\left[\Gamma(1+\hat{\rho}) \tilde{\hat{\phi}}\left(\frac{1}{\ell(\eta)}\right)\right]^{-1}>0 .
$$

(c) If $\boldsymbol{X}$ is infinitesimally efficient compared to $\hat{\boldsymbol{X}}$ and $Q^{+} \in(0,1)$ (and, thus, $\left.\rho=\hat{\rho}\right)$, then, for all $\varepsilon \in\left(0,1-Q^{+}\right)$, there exists an $\eta_{\varepsilon} \in(0,1)$ such that, for all $\eta \in\left(\eta_{\varepsilon}, 1\right]$,

$$
\mathrm{E}\left(\varepsilon\left(\eta, \eta^{\gamma}\right)\right)-\mathrm{E}\left(\hat{\mathrm{E}}\left(\eta^{\gamma}\right)\right)<\left(Q^{+}-1+\varepsilon\right) \hat{C}\left[\Gamma(1+\hat{\rho}) \tilde{\hat{\phi}}\left(\frac{1}{\ell(\eta)}\right)\right]^{-1}<0 .
$$

(d) If $\boldsymbol{X}$ is infinitesimally efficient compared to $\hat{\boldsymbol{X}}$ and $Q^{+}=Q=0$ (in particular, if $\hat{\rho}<\rho$ ), then, for all $\varepsilon \in(0,1)$, there exists an $\eta_{\varepsilon} \in(0,1)$ such that, for all $\eta \in\left(\eta_{\varepsilon}, 1\right]$,

$$
\begin{aligned}
\left(A_{\gamma}\right. & \left.-\hat{A}_{\gamma}-1-\varepsilon\right) \hat{C}\left[\Gamma(1+\hat{\rho}) \tilde{\hat{\phi}}\left(\frac{1}{\ell(\eta)}\right)\right]^{-1} \\
& <\mathrm{E}\left(\varepsilon\left(\eta, \eta^{\gamma}\right)\right)-\mathrm{E}\left(\hat{\mathrm{E}}\left(\eta^{\gamma}\right)\right) \\
& <\left(A_{\gamma}-\hat{A}_{\gamma}-1+\varepsilon\right) \hat{C}\left[\Gamma(1+\hat{\rho}) \hat{\hat{\phi}}\left(\frac{1}{\ell(\eta)}\right)\right]^{-1}
\end{aligned}
$$

(The quantities $A_{\gamma}$ and $\hat{A}_{\gamma}$ were defined in Lemma 5).

Moreover, if $\frac{1}{2} \geq \rho>\hat{\rho}$, there exists a $\gamma_{0} \in(1,2]$ such that, for all $\gamma \in\left(1, \gamma_{0}\right]$, we have $1-A_{\gamma}+\hat{A}_{\gamma}>0$ and, for all $\varepsilon \in\left(0,1-A_{\gamma}+\hat{A}_{\gamma}\right)$ and $\eta \in\left(\eta_{\varepsilon}, 1\right]$,

$$
\mathrm{E}\left(\mathscr{E}\left(\eta, \eta^{\gamma}\right)\right)-\mathrm{E}\left(\hat{\mathrm{E}}\left(\eta^{\gamma}\right)\right)<\left(A_{\gamma}-\hat{A}_{\gamma}-1+\varepsilon\right) \hat{C}\left[\Gamma(1+\hat{\rho}) \hat{\hat{\phi}}\left(\frac{1}{\ell(\eta)}\right)\right]^{-1}<0 .
$$

In all four cases, similar statements hold with $\hat{C}[\Gamma(1+\hat{\rho}) \tilde{\hat{\phi}}(1 / \ell(\eta))]^{-1}$ replaced by $\mathrm{E}(\hat{\mathrm{E}}(\eta))$.

Proof. Using (8) in Remark 2, we have, thanks to Lemmas 4 and 5,

$$
\begin{aligned}
Q^{-}-1+A_{\gamma}-\hat{A}_{\gamma} & \leq \liminf _{\eta \rightarrow 1^{-}} \frac{\mathrm{E}\left(\mathscr{E}\left(\eta, \eta^{\gamma}\right)\right)-\mathrm{E}\left(\mathscr{E}\left(1, \eta^{\gamma}\right)\right)}{\hat{C}(\ell(\eta))^{\hat{\rho}} \hat{L}(\ell(\eta))} \\
& \leq \limsup _{\eta \rightarrow 1^{-}} \frac{\mathrm{E}\left(\mathcal{E}\left(\eta, \eta^{\gamma}\right)\right)-\mathrm{E}\left(\mathscr{E}\left(1, \eta^{\gamma}\right)\right)}{\hat{C}(\ell(\eta))^{\hat{\rho}} \hat{L}(\ell(\eta))} \\
& \leq Q^{+}-1+A_{\gamma}-\hat{A}_{\gamma} .
\end{aligned}
$$


We then note that $\hat{L}(\ell(\eta))(\ell(\eta))^{\hat{\rho}}=[\Gamma(1+\hat{\rho}) \tilde{\hat{\phi}}(1 / \ell(\eta))]^{-1}$, and that the quantities $A_{\gamma}$ and $\hat{A}_{\gamma}$ are equal when $\rho=\hat{\rho}$. From this and the fact that $\hat{\mathrm{E}}\left(\eta^{\gamma}\right)=\mathcal{E}\left(1, \eta^{\gamma}\right)$ (cf. (7)), we readily deduce parts (a), (b), and (c) according to the values of $Q^{-}$and $Q^{+}$. For part (d), we use the previous arguments together with the last statement of Lemma 5.

The last assertion follows from the asymptotic equivalence (16).

Theorem 3 provides conditions on large thresholds $\eta$ and $\eta_{0}$ under which the use of the second fragmentation process can be determined to be efficient or not. We next briefly address the efficiency of using or not using the first fragmentation process. The arguments of the following theorem are similar to those of the previous lemmas, so we just sketch its proof. We use the following notation: for all $\gamma \in(1, \infty)$,

$$
B_{\gamma}:=\frac{\sin (\pi \rho)}{\pi} \int_{0}^{\gamma-1}(\gamma-1-u)^{\rho} \frac{\mathrm{d} u}{(1+u) u^{\rho}} .
$$

Theorem 4. (Two-step procedure versus first fragmentation only.) For all $\gamma \in(1, \infty)$, the following assertions hold.

(a) If $\hat{\boldsymbol{X}}$ is infinitesimally efficient compared to $\boldsymbol{X}$ then, for all $\varepsilon \in\left(0,1-1 / Q^{-}\right)$, there exists an $\eta_{\varepsilon} \in(0,1)$ such that, for all $\eta \in\left(\eta_{\varepsilon}, 1\right]$,

$$
\mathrm{E}\left(\varepsilon\left(\eta, \eta^{\gamma}\right)\right)-\mathrm{E}\left(\mathrm{E}\left(\eta^{\gamma}\right)\right)<\left(\frac{1}{Q^{-}}-1+\varepsilon\right) B_{\gamma} C\left[\Gamma(1+\rho) \tilde{\phi}\left(\frac{1}{\ell(\eta)}\right)\right]^{-1}<0 .
$$

(b) If $\boldsymbol{X}$ is infinitesimally efficient compared to $\hat{\boldsymbol{X}}$ and $Q^{+} \in(0,1)$ (and, thus, $\left.\rho=\hat{\rho}\right)$, then, for all $\varepsilon \in\left(0,1 / Q^{+}-1\right)$, there exists an $\eta_{\varepsilon} \in(0,1)$ such that, for all $\eta \in\left(\eta_{\varepsilon}, 1\right]$,

$$
\mathrm{E}\left(\mathcal{E}\left(\eta, \eta^{\gamma}\right)\right)-\mathrm{E}\left(\mathrm{E}\left(\eta^{\gamma}\right)\right)>\left(\frac{1}{Q^{+}}-1-\varepsilon\right) B_{\gamma} C\left[\Gamma(1+\rho) \tilde{\phi}\left(\frac{1}{\ell(\eta)}\right)\right]^{-1}>0 .
$$

(c) If $\boldsymbol{X}$ is infinitesimally efficient compared to $\hat{\boldsymbol{X}}$ and $Q^{+}=Q=0$ (in particular, if $\left.\hat{\rho}<\rho\right)$, then, for all $R>0$, there exists an $\eta_{R} \in(0,1)$ such that, for all $\eta \in\left(\eta_{R}, 1\right]$,

$$
\mathrm{E}\left(\mathcal{E}\left(\eta, \eta^{\gamma}\right)\right)-\mathrm{E}\left(\mathrm{E}\left(\eta^{\gamma}\right)\right)>R C\left[\tilde{\phi}\left(\frac{1}{\ell(\eta)}\right)\right]^{-1}>0
$$

In all cases, we can replace $C[\Gamma(1+\rho) \tilde{\phi}(1 / \ell(\eta))]^{-1}$ by $\mathrm{E}(\mathrm{E}(\eta))$.

Proof. Fix $\gamma>1$ and $\varepsilon \in(0,1)$. As in Lemma 5, we obtain, for all $y \in[0, \gamma-1]$,

$$
C(1-\varepsilon)^{2} y^{\rho} \leq \frac{\Psi(\ell(\eta) y)}{(\ell(\eta))^{\rho} L(\ell(\eta))} \leq C(1+\varepsilon)^{2} y^{\rho}
$$

and

$$
\hat{C}(1-\varepsilon)^{2} \frac{(\ell(\eta))^{\hat{\rho}} \hat{L}(\ell(\eta))}{(\ell(\eta))^{\rho} L(\ell(\eta))} y^{\hat{\rho}} \leq \frac{\hat{\Psi}(\ell(\eta) y)}{(\ell(\eta))^{\rho} L(\ell(\eta))} \leq \hat{C}(1+\varepsilon)^{2} \frac{(\ell(\eta))^{\hat{\rho}} \hat{L}(\ell(\eta))}{(\ell(\eta))^{\rho} L(\ell(\eta))} y^{\hat{\rho}}
$$

if $\eta$ is close enough to 1 . Now set $\bar{\partial}(\eta):=\mathrm{E}\left(\mathcal{E}\left(\eta, \eta^{\gamma}\right)-\mathcal{E}\left(\eta^{\gamma}, \eta^{\gamma}\right)\right)$. From the previous bounds, and from the explicit expression for $\bar{\partial}(\eta)$ given in Remark 2 , we deduce that

$$
C\left(\frac{A_{\gamma}}{Q^{+}}-B_{\gamma}\right) \leq \liminf _{\eta \rightarrow 1^{-}} \frac{\bar{\partial}(\eta)}{(\ell(\eta))^{\rho} L(\ell(\eta))} \leq \limsup _{\eta \rightarrow 1^{-}} \frac{\bar{\partial}(\eta)}{(\ell(\eta))^{\rho} L(\ell(\eta))} \leq C\left(\frac{A_{\gamma}}{Q^{-}}-B_{\gamma}\right) .
$$


Part (a) follows from this relation, using the facts that $A_{\gamma}=B_{\gamma}$ if $\rho=\hat{\rho}$ and $Q_{-}=\infty$ if $\hat{\rho}>\rho$. The remaining parts are similar.

Remark 6. If $\rho=\hat{\rho}$ and $Q \in(0, \infty)$ exists, we obtain, for $\eta$ close enough to 1 ,

$$
\begin{aligned}
& \mathrm{E}\left(\hat{\mathrm{E}}\left(\eta^{\gamma}\right)\right)+(Q-1-\varepsilon) \mathrm{E}(\hat{\mathrm{E}}(\eta))<\mathrm{E}\left(\varepsilon\left(\eta, \eta^{\gamma}\right)\right)<\mathrm{E}\left(\hat{\mathrm{E}}\left(\eta^{\gamma}\right)\right)+(Q-1+\varepsilon) \mathrm{E}(\hat{\mathrm{E}}(\eta)) \\
& \mathrm{E}\left(\mathrm{E}\left(\eta^{\gamma}\right)\right)+\left(Q^{-1}-1-\varepsilon\right) B_{\gamma} \mathrm{E}(\mathrm{E}(\eta))<\mathrm{E}\left(\mathcal{E}\left(\eta, \eta^{\gamma}\right)\right) \\
& \quad<\mathrm{E}\left(\mathrm{E}\left(\eta^{\gamma}\right)\right)+\left(Q^{-1}-1+\varepsilon\right) B_{\gamma} \mathrm{E}(\mathrm{E}(\eta))
\end{aligned}
$$

In particular, for $Q=1$, we deduce that $\mathrm{E}\left(\mathcal{E}\left(\eta, \eta^{\gamma}\right)\right) \sim \mathrm{E}\left(\hat{\mathrm{E}}\left(\eta^{\gamma}\right)\right) \sim \mathrm{E}\left(\mathrm{E}\left(\eta^{\gamma}\right)\right)$ when $\eta \rightarrow 1^{-}$, as one could expect.

\section{Acknowledgements}

J. Fontbona and S. Martínez are indebted to the Basal Conicyt Project, and N. Krell acknowledges support from the ARCUS project and Millennium Nucleus ICM P04-069-F. The authors thank an anonymous referee for valuable suggestions that allowed us to improve the presentation of this work.

\section{References}

[1] Berestycki, J. (2002). Ranked fragmentations. ESAIM Prob. Statist. 6, 157-175.

[2] Bertoin, J. (1996). Lévy Processes (Camb. Tracts Math. 121). Cambridge University Press.

[3] Bertorn, J. (1999). Subordinators: examples and applications. In Lectures on Probability Theory and Statistics (Saint-Flour, 1997; Lecture Notes Math. 1717), Springer. Berlin, pp. 1-91.

[4] Bertoin, J. (2001). Homogeneous fragmentation processes. Prob. Theory Relat. Fields 121, 301-318.

[5] Bertoin, J. (2006). Random Fragmentation and Coagulation Processes (Camb. Stud. Adv. Math. 102). Cambridge University Press.

[6] Bertoin, J. and Martínez, S. (2005). Fragmentation energy. Adv. Appl. Prob. 37, 553-570.

[7] Bertoin, J., van Harn, K. and Steutel, F. W. (1999). Renewal theory and level passage by subordinators. Statist. Prob. Lett. 45, 65-69.

[8] Hoffmann, M. ANd Krell, N. (2010). Statistical analysis of self-similar conservative fragmentation chains. To appear in Bernoulli.

[9] Kyprianou, A. E. (2006). Introductory Lectures on Fluctuations of Lévy Processes with Applications. Springer, Berlin.

[10] Walker, W. H., Lewis, W. K., McAdams, W. H. and Gilliland, E. R. (1937). Principles of Chemical Engineering. McGraw-Hill, New York. 